\title{
Combining multiple pairwise neural networks classifiers: a comparative study
}

\author{
Olivier Lezoray ${ }^{1}$ and Hubert Cardot $^{2}$ \\ ${ }^{1}$ LUSAC (EA 2607), groupe Vision et Analyse d'Image, IUT Dpt. SRC, 120 Rue de l'exode, \\ Saint-Lô, F-50000, France \\ Olivier. Lezoray@info.unicaen.fr \\ ${ }^{2}$ Université Francois-Rabelais de Tours, Laboratoire d'Informatique (EA 2101), 64 Avenue \\ Jean Portalis, Tours, F-37200, France \\ Hubert. Cardoteuniv-tours. fr
}

\begin{abstract}
Classifier combination constitutes an interesting approach when solving multiclass classification problems. We review standard methods used to decode the decomposition generated by a one-against-one approach. New decoding methods are proposed and are compared to standard methods. A stacking decoding is also proposed and consists in replacing the whole decoding by a trainable classifier to arbiter among the conflicting predictions of the binary classifiers. Substantial gain is obtained on all datasets used in the experiments.
\end{abstract}

\section{Introduction}

Since the advent of data mining in information management systems, the applications of multiclass pattern recognition has covered a very wide range including image or text categorization, object classification, speech recognition. Multiclass pattern recognition aims at building a function $F$ that maps the input feature space to an output space of more than two classes. Each example $(x, y)$ consists of an instance $x \in X$ and a label $y \in\{1, \ldots, K\}$ where $X$ is the feature vector input space and $K$ the number of classes to be discriminated. A general classifier can be considered as a mapping $F$ from instances to labels $F: X \rightarrow\{1, \ldots, K\}$. There are two ways for a classifier to solve multiclass problems: (1) consider all the data in one optimization problem, (2) construct several binary classifiers and combine them. The first approach formulates the multiclass problem into one single optimization problem (all-at-once). However the number of samples is the main factor that contributes to the time complexity for training the classifier, therefore algorithms of the first category are significantly slower that the ones that include several binary classifiers where each classifier classifies only a small portion of the data [1-4]. Moreover, muticlass classification is intrinsically harder than binary classification because the classification algorithm has to learn to construct a high number of separation boundaries whereas binary classifiers have to determine only one appropriate decision function. Currently, a common approach to construct a multiclass classifier relies in decomposing the multiclass problem into a set of binary ones and then combining their outputs to make a final multi-class prediction $[2,5]$. The basic idea behind combining binary classifiers is to decompose the multiclass problem into a set of easier and more accessible binary problems. The main advantage 
in this divide-and-conquer strategy is that any binary classification algorithm can be used. A binary classifier having to face with the classification of data using examples as positive ones and the others as negative ones, there are two schemes to construct several binary classifiers for multiclass ( $K$-class) problems [6]. The most traditional scheme (the standard method) [7] builds $K$ different classifiers. A separate concept is modeled by each classifier by defining a separate learning problem for each class. The original problem is split into a series of binary problems (one for each class) where the $i^{t h}$ classifier is trained while labeling all the samples in the $i^{t h}$ class as positive and the rest as negative. This technique is called one-against-all since each classifier separates one class from all the others. The drawbacks of this approach is that each binary classifier has to see all the training database and not a reduced version of it and the training data can be unbalanced which can distort each binary classifier. The second scheme constucts $K \times(K-1) / 2$ classifiers using all the pairwise combinations of the $K$ classes $[4,8,9]$. This technique is called one-against-one. The latter approach is very interesting since the binary decision not only contain fewer training examples but the decision function of each binary problem can be considerably simpler than in the case of one-against-all binarization since the classes have less overlap [1,2]. The major advantage of this approach is that it provides redundancy which can lead to better generalization abilities.

Beside choosing the way to decompose the problem, one also needs to devise a strategy for combining the binary classifiers and provide a final prediction, namely how to combine the outputs provided by all the binary classifiers. This is of importance to define a multiclass classifier from several binary ones. Whatever the used decomposition (one-against-all and one-against-one) if a simple voting strategy is used, there can be inconsistent regions [9] (less for one-against-one but still remain). For one-againstall the classifiers might all consider the input as not being of their class or several ones can conclude that it is of their class. For one-against-one, all the classifiers can disagree. The problem of combining binary classifiers has therefore been extensively studied and a lot of combining scheme have been proposed but many researchers reported opposing views to which scheme is better in terms of accuracy and speed [1,2,6-16]. Speed issues depend primarily on the different implementations of the basic binary classifier and accuracy issues depend on the nature of the basic learner, the data set and how the basic classifiers are well tuned to achieve maximum performance [7]. The litterature being inconclusive, the best method for combining binary classifiers is an important research issue which remains open. In this paper we propose to compare several classical combining schemes using multi layer perceptrons as the base learner, moreover we also propose new combining schemes which outperform the classical ones. We consider only the one-against-one formulation to proceed to the construction of binary neural networks. In section 2 we present the binary neural networks we used and in section 3 we discuss how to combine binary neural networks. In section 4 we demonstrate the effectiveness of the proposed combining methods by computer experiments. 


\section{Binary Neural Networks}

Since we consider the one-against-one decomposition scheme, for a classification problem with $K$ classes, a set of networks is built, each one being in charge of separating elements from two distinct classes. The set of different classes is denoted by $C=\left\{C_{1}, C_{2}, \ldots, C_{K}\right\}$ and $|C|=K$. For $K$ classes, that leads to have $(K \times(K-1)) / 2$ neural networks being used for classification (denoted as MLP for Multi Layer Perceptrons). The set of Binary Neural Networks (BNN) is given by $\mathcal{A}=\left\{\mathcal{R}_{C_{1}, C_{2}} ; \cdots ; \mathcal{R}_{C_{K-1}, C_{K}}\right\}$. The difficulty in separating $K$ classes all-at-once is simplified by the specialization of each network, because a network is interested only in the separation of two classes. When one of these neural networks learns how to differentiate two classes, only the objects belonging to these two classes are presented to the neural network. This implies, on the one hand to simplify the training (since the set of data to be learned is restricted) and on the other hand, to make easier the discrimination between these two classes since the network learnt how to recognize only those [17]. The global training dataset containing patterns of all the different classes is denoted by $D_{\text {Train }}$. The latter is divided in several subsets for each neural network. $D_{\text {Train }}\left(C_{i}, C_{j}\right)$ is the dataset which corresponds to the neural network which differenciates the classes $C_{i}$ and $C_{j}$ and contains patterns of only those two classes. The initial training data $\left(D_{\operatorname{Train}}\left(C_{i}, C_{j}\right)\right)$ associated to each neural network is split into two subsets: a learning set $\left(D_{\text {Learn }}\left(C_{i}, C_{j}\right)\right)$ and a validation set $\left(D_{V \text { alid }}\left(C_{i}, C_{j}\right)\right)$. The learning of a neural network is performed on $\left.D_{\text {Learn }} C_{i}, C_{j}\right)$ and the $D_{\text {Valid }}\left(C_{i}, C_{j}\right)$ validation set is used to evaluate the classification rate of the network during the training. Therefore the validation set is not used to learn the weights of neural networks, only to tune the hyperparameters (number of neurons, number of iterations, ...). The structure of the neural networks used is the following one: a layer of inputs containing as many neurons as the number of attributes associated with the object to be classified, a hidden layer containing a variable number of neurons and one output neuron. The value of the output neuron is in the interval ] $-1,1[$. According to the sign of the result associated with this single neuron, an object is classified in one of the two classes that the network separates. The neural networks used by our architecture are very simple (only one hidden layer, only one neuron of output). This has several advantages $[3,10,18]$. The simplicity of the task associated to each neural network simplifies the convergence of the training as well as the search for a simple structure. The generalization of their structure can be made in a dynamic way very easily. Therefore, an automatic method is used to find the number of hidden neurons that gives the best classification rate $[3,19,20]$. The output value provided by a BNN when a sample data $x$ has to be classified is denoted by $O\left(x, \mathcal{R}_{C_{i}, C_{j}}\right)$. From this output, $x$ can be classified as $C_{i}$ or $C_{j}$ according to the sign of the output: $x$ is considered as of class $C_{i}$ if $O\left(x, \mathcal{R}_{C_{i}, C_{j}}\right) \geq 0$ and $C_{j}$ otherwise. The output can therefore be directly used as an estimate for the class memberships. However it might be more suitable to have pairwise class probability estimates which can be obtained by [4] $r_{i j}(x)=\left(O\left(x, \mathcal{R}_{C_{i}, C_{j}}\right)+1\right) / 2$ and $r_{j i}(x)=1-r_{i j}(x) . r_{i j}(x)$ gives the pairwise posterior probability of the input vector $x$ to belong to the class $i$ and $r_{j i}(x)$ to the class $j$ (according to the single BNN $\mathcal{R}_{C_{i}, C_{j}}$ ). 


\section{Combining binary classifiers}

Constructing multiclass classifiers from a pairwise decomposition consists in combining the $B=(K \times(K-1) / 2)$ pairwise classifiers outputs. Each binary classifier is a mapping $f_{b}: X \rightarrow \mathbb{R}$ with $b \in\{1, \ldots, B\}$. A vector $f(x)=\left(f_{1}(x), \ldots, f_{B}(x)\right)$ is constructed from the outputs of the binary classifiers. A combination rule $g$ can then be applied to combine all the outputs $f(x)=\left(f_{1}(x), \ldots, f_{B}(x)\right)$ using a function $g: \mathbb{R}^{B} \rightarrow \mathbb{R}^{K}$ which couples the estimates of each binary classifier in order to obtain class membership estimates (which can be probabilities) for the multi-class problem. Once the class memberships $\mu$ have been estimated $g(f(x))=g\left(f_{1}(x), \ldots, f_{B}(x)\right)=$ $\left(\mu\left(C_{1} \mid x\right), \ldots, \mu\left(C_{K} \mid x\right)\right)$, a final selection scheme is used to choose the winner class. This is done as a mapping $h: \mathbb{R}^{K} \rightarrow\{1, \ldots, K\}$. The whole $\mathrm{K}$-ary classifier combining all the binary classifiers scores (obtained by pairwise decomposition) is denoted by $F(x)=h(g(f(x)))$ where $h$ is the selection scheme function applied to select the winner class ang $g$ the combination rule. $h \circ g$ defines the complete decoding scheme needed to perform multiclass classification from binary pairwise classifiers.

\subsection{Standard Decoding}

To obtain class membership estimates from the BNN outputs, a combination rule $g$ is needed to perform the decoding. This rule associates a vector of BNN outputs $f(x)$ with a vector of class membership estimates $g(f(x))=\left(\mu\left(C_{1} \mid x\right), \ldots, \mu\left(C_{K} \mid x\right)\right)$. In this section we review the classical combination rules that can be used to that aim and propose new ones.

Majority vote The most commonly used combination rule is probably the Majority Vote (MV) one. With this combination rule [21], each class receives votes from individual classifiers. The membership estimates correspond to the number of votes received by each class. $\mu\left(C_{i} \mid x\right)=\Sigma_{j} V\left(r_{i j}(x) \geq 0.5\right)$ with $V(x)=1$ if $x$ is true and 0 otherwise. The chosen class is the one which receives the largest number of votes) and $h=\operatorname{argmax}$.

Hastie A way to obtain class membership estimates from the pairwise probability estimates $r_{i j}(x)$ has been proposed by Hastie [8]. To combine all the estimates of the BNNs we would like to obtain a set of class membership probabilities $p_{i}(x)=P\left(C_{i} \mid x\right)$. The $r_{i j}$ are related to the $p_{i}$ according to $r_{i j}(x)=\frac{p_{i}(x)}{p_{i}(x)+p_{j}(x)}$. In order to find the best approximation $r_{i j}^{\prime}=\frac{\mu_{i}(x)}{\mu_{i}(x)+\mu_{j}(x)}$ the algorithm starts with $\mu_{i}(x)=\frac{2 \Sigma_{j \neq i} r_{i j}(x)}{K(K-1)}$ and computes the corresponding $r_{i j}^{\prime}(x)$. The $\mu_{i}(x)$ are obtained by minimizing the average Kullback-Leibler distance between $r_{i j}(x)$ and $r_{i j}^{\prime}(x)$. At convergence we have the class membership estimates with $\mu\left(C_{i} \mid x\right)=\mu_{i}(x)$. The winner class is considered as the most likely one and $h=\operatorname{argmax}$.

Price Another approach for estimating the class memberships has been proposed by Price [4]. It is based on the fact that neural networks trained to minimize a MSE 
cost function estimate posterior probabilities. A BNN with sigmoidal transfer function can compute the posterior probabilities for the two classes (previously denoted by $r_{i j}(x)$ and $r_{j i}(x)$ ). One can then obtain the final expression of the class membership by: $\mu\left(C_{i} \mid x\right)=\frac{1}{\sum_{j \neq i} \frac{1}{r_{i j}(x)}-(K-2)}$. As for the Hastie combination rule, we have $h=\operatorname{argmax}$.

ECOC Another interesting combination rule is based on Error Correcting Output Codes (ECOC) [11,22]. It has been introduced to combine the outputs of binary Support Vector Machines. For a problem with $K$ classes, it creates a matrix $M \in$ $\{-1,0,1\}^{K \times B}$. A column in the matrix $M$ corresponds to a binary classifier $\mathcal{R}_{i, j}$ and a row corresponds to a class. For instance the first column corresponds to the classifier $\mathcal{R}_{1,2}$ and it learns to recognize the classes 1 and 2 (respectively the +1 and -1 coefficients of the column, the other coefficients are set to 0 since the classifier does not differentiate the other classes). Combining all the binary classifiers to estimate the class memberships consists in comparing the matrix rows with the classifiers outputs expressed by : $\mu\left(C_{k} \mid x\right)=\sum_{b=1}^{B} L\left(M(k, b) . f_{b}(x)\right)$. This provides the distortion between the vector of the BNN outputs $f(x)$ and the row $M(k, \cdot)$. For this combination rule the outputs of the binary classifiers $O\left(x, \mathcal{R}_{i j}\right)$ are directly used and not the $r_{i j}(x)$. We used Loss Based decoding and have set $L(z)=\exp (-z)$. For ECOC decoding schemes $h=$ argmin since this leads to find the row being the most similar to the classifiers outputs $[23,24]$.

Min-Max For all the previous decoding schemes, the probability estimates of the classifiers obtained by the combination rule $g$ are used to assign to an input pattern the class with the maximal output. Combining all the pairwise classifiers can lead to bad results since if the input $x$ is of class $C_{i}$, there are only $(K-1)$ relevant classifiers among the $(K \times(K-1)) / 2$ which have seen the class $C_{i}$ and the remaining $((K-1) \times(K-2)) / 2$ irrelevant classifiers have never seen inputs from class $C_{i}$. While classifying an input one whishes that relevant classifiers will provide coherent informations to cope with all the irrelevant ones. To try to alleviate this problem, we propose to get the minimum value of $r_{i j}(x)$ for each class $C_{i}: \mu\left(C_{i} \mid x\right)=\min _{j}^{K-1} r_{i j}(x)$. Finally we select the class which maximizes this minimum value: $h=\operatorname{argmax}$. The principle of this method consist in choosing the candidate class whose probability is less bad than that for all other candidate classes. The intuitive idea behind is that having a high pairwise probability for a particular pair of classes does not imply a strong decision towards this class because of irrelevant classifiers. However it can be rejected if the probability is low.

\subsection{Elimination Decoding}

Another decoding scheme is the elimination decoding one. This decoder was originally described by Kressel [5] and reintroduced by Platt [12] where it was called Directed Acyclic Graph (DAG). One strong argument for using DAG is that it resolves the problem of unclassifiable regions for pairwise classification. The elimination decoding is nothing more than a decision-tree based pairwise classification. Two sets are used 
where |.| stands for $\operatorname{Card}($.$) . The set of binary classifiers \mathcal{A}_{0}=\left\{\mathcal{R}_{1,2}, \ldots, \mathcal{R}_{K-1, K}\right\}$ (with $\left|\mathcal{A}_{0}\right|=B$ ) contains all the binary classifiers and the set $E_{0}=\left\{C_{1}, \ldots, C_{K}\right\}$ (with $\left|E_{0}\right|=K$ ) contains all the candidate winner classes. Elimination decoding operates iteratively. At each iteration $t=\{1, \ldots, K-1\}$, the size of $E_{t}$ is decreased by one (one class $C_{k}$ is eliminated) and all the classifiers discriminating $C_{k}$ in $\mathcal{A}_{t}$ are eliminated [24]: $\mathcal{A}_{t+1}=\mathcal{A}_{t}-\left\{\mathcal{R}_{i, j}: i=C_{k} \vee j=C_{k}\right\}$. The set $\mathcal{A}_{K-2}$ contains only one binary classifier which determines the winner class. Several problems occur however when using DAGs. First of all the choice of the winner class depends on the sequence of binary classifiers in nodes which affects the reliability of the algorithm. Moreover the correct class to be predicted is more or less advantaged according to its distance to the root node (higher risk of being rejected in the nodes near the root). Secondly since there are a lot of classifiers which are irrelevant for a given classification, using these classifiers can cause severe defects. To overcome this problem, several authors have proposed to use an adaptative DAG by optimizing its structure, however the generalization ability still depends on the structure of the tree [10,18, 25-27]. We propose a new elimination decoding which takes into account all the outputs of the binary classifiers. When using a classical decoding scheme without elimination, one selects the class with the largest probability ( $h=\operatorname{argmax}$ ). In the case of elimination, we want to eliminate the least credible class and this comes back to eliminate the class having the minimum of probability. The class to be eliminated is deduced from the class membership estimates : $C_{k}=h(g(f(x))) . g$ can be anyone of the previous combination rules (MV, Price, Hastie, Max) and since the method eliminates the least probable class at each iteration, we have $h=\operatorname{argmin}$. At the iteration $t$, the number of candidate classes is $\left|E_{t}\right|=(K-t)$ and the number of binary classifiers to be combined is $\left|\mathcal{A}_{t}\right|=(K-t)(K-t-1) / 2$. This new elimination decoding is different of the Direct Acyclic Graph [12] since at each iteration, all the outputs of candidate binary classifiers are combined to determine the class to be eliminated. Whereas with a DAG, only one classifier output is used for eliminating a class at each iteration.

\subsection{Staking Decoding}

The combination of the class membership estimates can be performed via a separate trainable classifier [23,28]. This classifier is seen as a Meta Classifier fed by the output vector $f(x)$ of all the binary classifiers. This method is also referred to as stacking [29]. This approach seems more suitable than all the previous ones for the following reason. As said before, combining classifiers which have never seen instances from one same class during the training phase results in combining different information sources. The combination of these ignorant classifiers (a binary classifier has seen only two classes among $K$ ) with respect to the others can therefore result in almost random classification. Indeed decoding methods such as voting rely on the assumption that the relevant classifiers mainly predict the correct class and provide more votes to the true class than the irrelevant classifiers to any other class. However if some of the relevant classifiers predict wrong classes, the final classification can be also wrong. Since we cannot predict the behavior of irrelevant classifiers, more sophisticated decoding schemes are needed. To that aim, a trainable classifier is used with as training input the output vector $f(x)$ of all the binary classifiers. Each feature vector $x_{i},(i=1, \ldots, N)$ 
of the training set $D_{\text {Train }}$ is used to feed all the binary classifiers. A new example $\left.\left(f\left(x_{i}\right), y_{i}\right)\right)$ is then obtained. Such a process can be repeated so that a new training set $D_{\text {Train }}^{\prime}=\left\{\left(f\left(x_{1}\right), y_{1}\right), \ldots,\left(f\left(x_{N}\right), y_{N}\right)\right\}$ is generated. The new training data set is used to train a Meta Classifier which predicts the final class by $F(x)=h(g(f(x)))$. The function $h \circ g$ designs the Meta Classifier to be used. The $D_{\text {Train }}^{\prime}$ database generated by all the binary classifiers provides valuable information about the possible misleading predictions caused by the irrelevant classifiers. The potential gain of stacking for decoding is evident and it can lead to correct predictions where other methods would fail $[28,30]$.

\section{Experimental results}

This section presents an experimental comparison of the ways to combine binary neural networks according to the different combining rules. The databases for which results will be presented here are data bases coming from the Machine Learning Data Repository of the University of California at Irvine (UCI) [31]. Table 1 describes the different databases showing the variety of training data set sizes $\left(\left|D_{\text {Train }}\right|\right)$, the number of classes $(K)$, the number of neural networks $(B)$ and the dimensionality of the data input $(|x|)$. The tests are performed on a data set $\left(D_{\text {Test }}\right)$ independent of the training set. Table 2 presents all the classification rates obtained on $D_{T e s t}$ for the different

\begin{tabular}{lrrrrr}
\hline Database & $K\left|D_{\text {Train }}\right|\left|D_{\text {Test }}\right||x|$ & $B$ \\
\hline Iris & 3 & 120 & 30 & 4 & 3 \\
Wine & 3 & 144 & 34 & 13 & 3 \\
Vehicle & 4 & 679 & 167 & 18 & 6 \\
PageBlocks & 5 & 4382 & 1091 & 10 & 10 \\
SatImage & 6 & 4435 & 2000 & 36 & 15 \\
Shuttle & 7 & 43500 & 14500 & 9 & 21 \\
PenDigits & 10 & 7494 & 3498 & 16 & 45 \\
OptDigits & 10 & 3065 & 760 & 64 & 45 \\
Letter & 26 & 16000 & 4000 & 16 & 325 \\
\hline
\end{tabular}

Table 1. Data bases used for the tests.

combining rules (best rates bold faced for each decoding rule family). For the standard decoding, the results are homogeneous, except for Hastie and Price methods which perform significantly worse on several datasets. As expected from the litterature, ECOC decoding performs very well and provides results always better than the Majority Vote. One thing to point out with ECOC is that it can be a robust combining method as long as the errors of the binary classifiers are not correlated $[13,15]$. For this purpose all the dichotomies must be as dictinct as possible, using well-tuned binary classifiers does the matter and explains why ECOC works well in our study. When ECOC is not best combining method, best results are obtained with the proposed Min-Max method which 


\begin{tabular}{|l|r|r|r|r|r|r|r|r|r|}
\hline & Iris & Wine & Vehicle & PageBlocks & SatImage & Shuttle & Pendigits & Optdigits & Letter \\
\hline \multicolumn{8}{|c|}{ all-at-once } \\
\hline MLP & 70.00 & 97.06 & 66.67 & 84.80 & 80.00 & 79.15 & 83.82 & 90.39 & 62.45 \\
\hline \multicolumn{8}{|c|}{ one-against-one standard decoding } \\
\hline Majority vote & $\mathbf{7 0 . 0 0}$ & $\mathbf{9 7 . 0 6}$ & 69.46 & 88.27 & 77.90 & 95.70 & 89.17 & 91.70 & 78.37 \\
\hline Hastie & 63.33 & $\mathbf{9 7 . 0 6}$ & 68.26 & 42.35 & $\mathbf{8 0 . 1 0}$ & 95.01 & 82.19 & 81.69 & 65.50 \\
\hline Price & 63.33 & $\mathbf{9 7 . 0 6}$ & $\mathbf{7 0 . 0 6}$ & 46.29 & 79.90 & 95.18 & 88.42 & 87.62 & 71.57 \\
\hline Ecoc & $\mathbf{7 0 . 0 0}$ & $\mathbf{9 7 . 0 6}$ & 69.46 & $\mathbf{8 8 . 4 5}$ & $\mathbf{8 0 . 1 0}$ & $\mathbf{9 5 . 8 2}$ & 89.05 & 90.91 & $\mathbf{7 8 . 5 2}$ \\
\hline Min-Max & $\mathbf{7 0 . 0 0}$ & $\mathbf{9 7 . 0 6}$ & 69.46 & 88.36 & 78.35 & 95.57 & $\mathbf{8 9 . 2 2}$ & $\mathbf{9 1 . 8 3}$ & 77.72 \\
\hline \multicolumn{8}{|c|}{ one-against-one elimination decoding } \\
\hline Majority vote & $\mathbf{7 0 . 0 0}$ & $\mathbf{9 7 . 0 6}$ & 68.26 & 88.36 & 77.85 & 95.66 & 89.19 & 91.04 & 78.44 \\
\hline Hastie & $\mathbf{7 0 . 0 0}$ & $\mathbf{9 7 . 0 6}$ & 68.86 & 88.36 & 78.20 & 95.63 & 89.14 & 91.17 & 77.54 \\
\hline Price & $\mathbf{7 0 . 0 0}$ & $\mathbf{9 7 . 0 6}$ & 68.86 & $\mathbf{8 8 . 4 5}$ & 78.10 & 95.62 & 89.11 & 91.17 & 77.64 \\
\hline Ecoc & $\mathbf{7 0 . 0 0}$ & $\mathbf{9 7 . 0 6}$ & 68.86 & $\mathbf{8 8 . 4 5}$ & 78.20 & $\mathbf{9 5 . 7 6}$ & 89.17 & 91.17 & $\mathbf{7 8 . 4 7}$ \\
\hline Max-Min & $\mathbf{7 0 . 0 0}$ & $\mathbf{9 7 . 0 6}$ & $\mathbf{6 9 . 4 6}$ & 88.36 & $\mathbf{7 8 . 3 5}$ & 95.59 & $\mathbf{8 9 . 2 8}$ & $\mathbf{9 1 . 7 0}$ & 77.67 \\
\hline \multicolumn{8}{|c|}{ Stacking decoding } \\
\hline C4.5 & $\mathbf{9 0 . 0 0}$ & $\mathbf{1 0 0 . 0 0}$ & $\mathbf{7 5 . 4 0}$ & $\mathbf{9 2 . 3 0}$ & $\mathbf{8 5 . 1 0}$ & $\mathbf{9 9 . 8 0}$ & $\mathbf{9 3 . 1 0}$ & $\mathbf{9 3 . 0 2}$ & $\mathbf{8 1 . 0 0}$ \\
\hline
\end{tabular}

Table 2. Classification rates of the different decoding methods.

confirms the intuitive idea that in some cases the irrelevant classifiers have strong influence on the final decision. Another advantage of the Min-Max method is its simplicity. One can therefore say that even if the results are very mixed, two standard decoding schemes can be retained as the best ones : ECOC and the proposed Min-Max method. If we have a look now to the results obtained with the proposed elimination decoding method, the first interesting thing is that the results look much more homogeneous between the different combining rules. As it was noted by Platt with DAGs, using an iterative elimination method reduces the error bound [12] since it avoids the problem of irrelevant regions of classification. However the elimination method we propose performs in general better than classical DAGs [14], proving that using an elimination method based on combining rules is a more robust method than one based on a decision tree of binary classifiers, whitout the problem of optimizing the structure of the tree. This is all the more interesting since our elimination decoding reduces the error bound whatever the combining rule. As for the standard decoding method the two best combining rules are ECOC and Max-Min (since we perform an elimination we do not have Min-Max : at each iteration the class minimizing the highest pairwise probability is eliminated). Finally we analyze the results of the proposed stacking decoding. First of all one has to note that using a meta-classifier for stacking implies to cope with quite large problems. For example for the 26-class Letter dataset, there are $26 \times 25=650$ predictions for each of 16000 examples. We have used decision trees (C4.5 [32]) metaclassifiers to perform stacking. The stacking meta-classifier is fed with the input vector $f(x)$ of all the predictions provided by the binary classifiers. On all datasets a 10-fold cross validation is performed. It can be seen that stacking decoding always give the best results on all the datasets. For several of them very significant improvements over all the other decoding methods are obtained. As compared to the work in Savicky [28], the 
use of posterior probabilities instead of hard class decisions of the binary classifiers to feed the stacking meta classifier enables substantial gain in the recognition rate. Simple meta-classifier such as decision tree which are linear classifier are sufficient to obtain better results than with a classical all-at-once MLP approach as seen in Table 2. Using binary classifiers is therefore very interesting since it can be viewed as an ensemble method which performs a simplification of the problem by decomposing it, the latter results been easier to classify by stacking than the initial ones all-at-once.

\section{Conclusion}

In this paper we reviewed and evaluated classical methods for multiclass classification based on binary neural networks according to the one-against-one formalism. We have also introduced a new standard decoding method (Min-Max) and a new elimination decoding which are both as suitable as the classical methods presented in the litterature as proved by the experiments. We also evaluated a technique using stacking decoding where the basic idea is to replace the combining and selection rules by a single metaclassifier that combines all the predictions of the binary classifiers. The training set of the meta-classifier consists of all the predictions of the binary classifiers for each training sample. Using stacking decoding leads to substential gain in the recognition rate. Future work will concern the use of the set of one-against-one classifiers as a new input sample generator [30] to increase the size of the training dataset of the meta classifier when the latter is unbalanced, preliminary results having also shown a new gain in the recognition rate.

\section{References}

1. Furnkranz, J.: Round robin classification. Journal of Machine Learning Research 2 (2002) 721-747

2. Furnkranz, J.: Pairwise classification as an ensemble technique. In: European Conference on Machine Learning (ECML). (2002) 97-110

3. Lezoray, O., Cardot, H.: A neural network architecture for data classification. International Journal of Neural Systems 11 (2001) 33-42

4. Price, D., Knerr, S., Personnaz, L.: Pairwise neural network classifiers with propabilistic outputs. In: Advances in Neural Information Processing Systems (NIPS). Volume 7., MIT Press (1995) 1109-116

5. Kressel, U.: Pairwise classification and support vector machines. In: Advances in Kernel Methods, Support Vector Learning. MIT Press (1999)

6. Ou, G., Murphey, Y., Feldkamp, A.: Multiclass pattern classification using neural networks. In: International Conference on Pattern Recognition (ICPR). Volume 4. (2004) 585- 588

7. Rifkin, R., Klautau, A.: In defense of one-vs-all classi.cation. Journal of Machine Learning Research 5 (2004) 101-141

8. Hastie, T., Tibshirani, R.: Classification by pairwise coupling. The annals of Statistics $\mathbf{2 6}$ (1998) 451-471

9. Tax, D., Duin, R.: Using two-class classifiers for multiclass classification. In: International Conference on Pattern Recognition (ICPR). Volume 2. (2002) 124-127

10. Lezoray, O., Fournier, D., Cardot, H.: Neural network induction graph for pattern recognition. Neurocomputing (2004) 257-274 
11. Allwein, E., Schapire, R., Singer, Y.: Reducing multiclass to binary: a unifying approach for margin classifiers. Journal of Machine Learning Research 1 (2000) 113-141

12. Platt, J., Cristianini, N., Shawe-Taylor, J.: Large margin dags for multiclass classification. In: Advances in Neural Information Processing Systems (NIPS). Volume 12., MIT Press (2000) 547-553

13. Moreira, M., Mayoraz, E.: Improved pairwise coupling classification with correcting classifiers. In: European Conference on Machine Learning (ECML), Springer-Verlag (1998) $160-171$

14. Hsu, C.W., Lin, C.J.: A comparision of methods for multi-class support vector machines. IEEE Transactions on Neural Networks 13 (2002) 415-425

15. Mayoraz, E., Alpaydin, E.: Support vector machines for multi-class classification. In: International Work conference on Artificial Neural Networks. Volume 2. (1999) 833-842

16. F. Tahahashi, S.A.: Optimizing directed acyclic graph support vector machines. In: Artificial Neural Networks in Pattern Recognition (ANNPR). (2003)

17. Lu, B.L., Ito, M.: Task decomposition and module combination based on class relations: A modular neural network for pattern classification. IEEE Transaction on Neural Networks $\mathbf{1 0}$ (1999) 1244-1256

18. Cardot, H., Lezoray, O.: Graph of neural networks for pattern recognition. In: International Conference on Pattern Recognition (ICPR). Volume 2. (2002) 124-127

19. Campbell, C.: Constructive Learning Techniques for Designing Neural Network Systems. San Diego: Academic Press (1997)

20. Kwok, T.Y., Yeung, D.Y.: Constructive algorithms for structure learning in feedforward neural networks for regression problems. IEEE Trans. on Neural Networks 8 (1997) 630 645

21. Friedman, J.: Another approach to polychotomous classification. Technical report, Dept. of statistics, Stanford University (1996)

22. Crammer, K., Singer, Y.: On the learnability and design of output codes for multiclass problems. Machine Learning 47 (2002) $201-233$

23. Klautau, A., Jevtić, N., Orlitsky, A.: Combined binary classifiers with applications to speech recognition. In: International Conference on Spoken Language Processing (ICSLP). (2002) $2469-2472$

24. Klautau, A., Jevtić, N., Orlitsky, A.: On nearest neighbor error-correcting output codes with application to all-pairs multiclass support vector machnies. Journal of Machine Learning Research 4 (2003) 1-15

25. Ko, J., Kim, E., Byun, H.: Improved n-division output coding for multiclass learning problems. In: International Conference on Pattern Recognition (ICPR). Volume 3. (2004) 470473

26. Phetkaew, T., Kijsirikul, B., Rivepiboon, W.: Reordering adaptive directed acyclic graphs: an improved algorithm for multiclass support vector machines. In: International Joint Conference on Neural Networks (IJCNN). Volume 2. (2003) 1605- 1610

27. Vural, V., Dy, J.G.: A hierarchical method for multi-class support vector machines. In: International Conference on Machine Learning (ICML). (2004)

28. Savicky, P., Furnkranz, J.: Combining pairwise classifiers with stacking. In: Intellignent Data Analysis (IDA). (2003)

29. Wolpert, D.: Stacked generalization. 'Neural Networks 5 (1992) 241-260

30. Zhou, Z.H.: Nec4.5: Neural ensemble based c4.5. IEEE Transactions on Knowledge and Data Engineering (2003)

31. S. Hettich, C.B., Merz, C.: UCI repository of machine learning databases. Technical report, University of California, Irvine, Dept. of Information and Computer Sciences (1998)

32. Quinlan, J.: C4.5 : programs for machine learning. Morgann Kauffman, San Mateo (1993) 